\title{
Flickering as an early warning signal
}

\author{
Vasilis Dakos • Egbert H. van Nes • Marten Scheffer
}

\begin{abstract}
Most work on generic early warning signals for critical transitions focuses on indicators of the phenomenon of critical slowing down that precedes a range of catastrophic bifurcation points. However, in highly stochastic environments, systems will tend to shift to alternative basins of attraction already far from such bifurcation points. In fact, strong perturbations (noise) may cause the system to "flicker" between the basins of attraction of the system's alternative states. As a result, under such noisy conditions, critical slowing down is not relevant, and one would expect its related generic leading indicators to fail, signaling an impending transition. Here, we systematically explore how flickering may be detected and interpreted as a signal of an emerging alternative attractor. We show that - although the two mechanisms differ-flickering may often be reflected in rising variance, lag-1 autocorrelation and skewness in ways that resemble the effects of critical slowing down. In particular, we demonstrate how the probability distribution of a flickering system can be used to map potential alternative attractors and their resilience. Thus, while flickering systems differ in many ways from the classical image of critical transitions, changes in their dynamics may carry valuable information about upcoming major changes.
\end{abstract}

Electronic supplementary material The online version of this article (doi:10.1007/s12080-013-0186-4) contains supplementary material, which is available to authorized users.

\author{
V. Dakos $(\square)$ \\ C/ Américo Vespucio S/N, \\ 41092 Seville, Spain \\ e-mail: vasilis.dakos@gmail.com \\ E. H. van Nes $\cdot$ M. Scheffer \\ The Netherlands \\ E. H. van Nes \\ e-mail: egbert.vannes@wur.nl \\ M. Scheffer \\ e-mail: marten.scheffer@wur.nl
}

Integrative Ecology Group, Estación Biológica de Doñana,

Department of Aquatic Ecology \& Water Quality Management, Wageningen University, PO Box 47, 6700AA Wageningen,
Keywords Resilience · Critical transition · Critical slowing down $\cdot$ Alternative stable states $\cdot$ Regime shift $\cdot$ Stochasticity

\section{Introduction}

Theory predicts that, close to tipping points, the return to equilibrium upon small perturbations will slow down (Wissel 1984; Strogatz 1994; Scheffer et al. 2009). In stochastic environments, this will tend to be reflected in higher temporal autocorrelation and variance of the fluctuations in the state of a system (Held and Kleinen 2004; Carpenter and Brock 2006; Scheffer et al. 2009). However, this picture of a critically slowed down world prior to a transition could be the exception rather than the rule. As most systems are embedded in highly stochastic environments, they may start to "flicker" between the basins of attraction of their potential alternative states far before bifurcation points at which critical transitions occur. Under such noisy conditions, one should therefore fail to observe the gradual climax of critical slowing down and would not expect its related generic leading indicators, signaling the approaching transition (Contamin and Ellison 2009; Scheffer et al. 2009; Carpenter and Brock 2010; Perretti and Munch 2012).

Indeed, many studies that provide empirical evidence of critical slowing down have been derived from controlled experiments in the lab where a system was studied under almost noise-free conditions. In these cases, a system was slowly pushed towards a tipping point, and critical slowing down was identified either directly by perturbation experiments (Veraart et al. 2012, Fig. 1a), or indirectly by estimating generic indicators, such as increased variance and autocorrelation, while comparing them to a control treatment (Drake and Griffen 2010, Fig. 1b). Such noise-free time series, however, are rare when derived from empirical observations or from field experiments. For example, paleoreconstructed isotope data of abrupt shifts between warming and cooling episodes in the past 60,00 years, known as Dansgaard-Oeschgaard events (DO event, Dansgaard et al. 
1993), have been hypothesized to represent stochastically induced jumps between alternative attractors (Ganopolski and Rahmstorf 2002; Ditlevsen and Johnsen 2010, Fig. 1c). In addition, a whole-lake manipulation experiment (Carpenter et al. 2011) suggested that planktivorous fish abundance exhibited short excursions to the alternative state a year before the final shift to piscivore dominance (Fig. 1d).

Although such behavior (Fig. 1c, d) would prevent the detection of critical slowing before an approaching transition (Scheffer et al. 2009), it will also affect indicators such as temporal autocorrelation and variance. Not surprisingly, variance will rise as flickering occurs (Carpenter and Brock 2006; Carpenter et al. 2008; Wang et al. 2012). However, temporal autocorrelation may either increase (Dakos et al. 2012a; Lenton et al. 2012) or decrease when resolution of the time series is low (Wang et al. 2012). Moreover, under extremely stochastic conditions or even chaotic dynamics (Hastings and Wysham 2010), neither variance nor autocorrelation may signal approaching transitions (Contamin and Ellison 2009; Cimatoribus et al. 2012; Perretti and Munch 2012). In a recent summary of available methods for detecting critical transitions in time series, Dakos et al. (2012a) have demonstrated that, for highly stochastic systems, upcoming transitions may be better
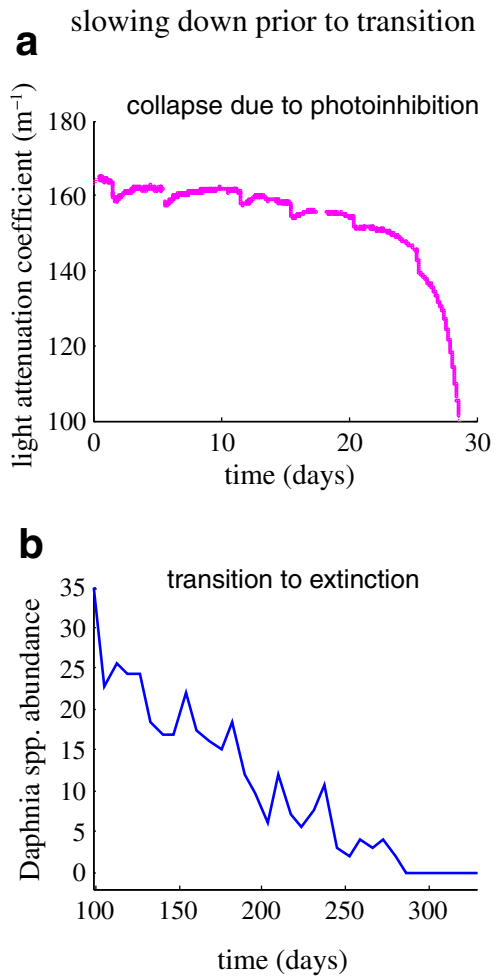

Fig. 1 Typical time series that provided empirical evidence of critical slowing down are derived from controlled experiments under a practically noise-free setting: a critical slowing down was identified directly by a perturbation experiment in a plankton chemostat (Veraart et al. 2012); b generic indicators, variance, autocorrelation and skewness, were compared to a control treatment in a zooplankton experiment (Drake and Griffen 2010). Instead, empirical observations or field detected using nonparametric models (Carpenter and Brock 2011) or threshold time-varying autoregressive models (Ives and Dakos 2012). Overall, however, the consequences of flickering on the detection of critical transitions are still not completely resolved.

Here, we systematically explore how flickering affects the performance of generic early warning signals for critical transitions. We compare variance, autocorrelation, and skewness estimated in situations with flickering versus situations with critical slowing down prior to a transition. In particular, we show how patterns in generic indicators and probability distributions shaped by flickering can be suitable search images when looking for fingerprints of approaching critical transitions in ecological time series derived from observations in the field.

\section{Methods}

\section{Model description}

We used a minimal model that describes the shift of a lake from an oligotrophic to a eutrophic state (Carpenter et al.

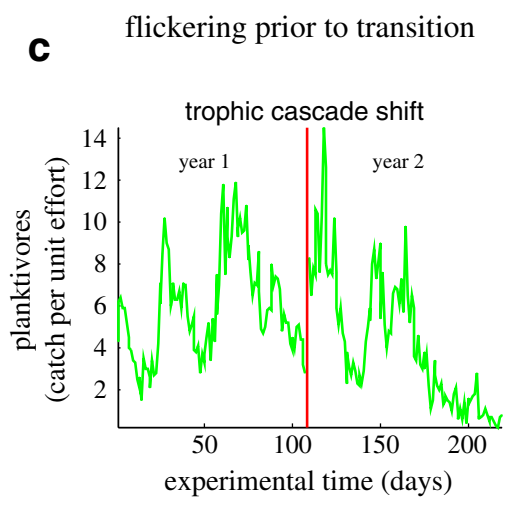

d

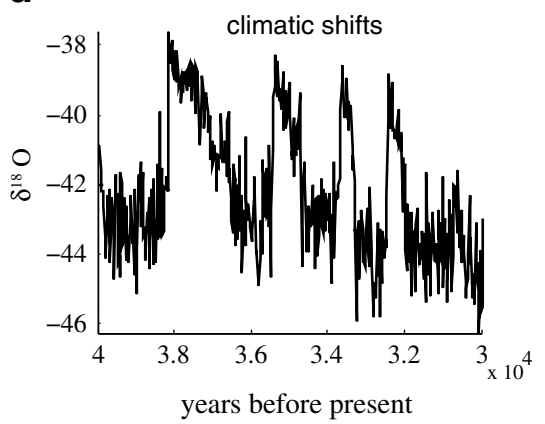

experiments appear more like sudden occasional jumps between alternative attractors: c paleo-reconstructed isotope data of abrupt shifts between warming and cooling episodes in the past 60,00 years, known as Dansgaard-Oeschgaard events (Svensson et al. 2008); d induced trophic cascade in a lake experiment (Carpenter et al. 2011) exhibited rapid short excursions of planktivore fish to the alternative state before the shift 
1999). The model has been previously used to study critical transitions and their precursors (Guttal and Jayaprakash 2008; Dakos et al. 2010). In the model (Eq. 1), phosphorus dynamics $P$ are determined by gains from the surrounding catchment with input rate $\alpha$ and by losses to the lake sediment and flushing with rate $s$. At low phosphorus input rates, the lake remains oligotrophic, but when input loading increases, phosphorus is recycled strongly from the sediment back to the water column with maximum rate $r$ and the lake may suddenly become eutrophic. Thus, the model reads:

$\mathrm{d} P=\left(\alpha-s P+r \frac{P^{8}}{P^{8}+1}\right) \mathrm{d} t+\sigma P \mathrm{~d} W$

where to the deterministic processes, we also added stochastic disturbances in the form of white noise $\mathrm{d} W$ with magnitude $\sigma$. In particular, we scaled this magnitude to the actual level of phosphorus concentration $P$. This means that disturbances to phosphorus dynamics were proportional to the levels of phosphorus in the lake, an assumption that reflects a real case scenario. This formulation also does not specify the sources of disturbances (be either through input to the lake, recycling or purely unrelated stochastic events). Technically, this multiplicative noise prevents the model from reaching unrealistic negative phosphorus concentrations due to a strong noise regime and increases the probability of the system to escape to the alternative attractor even at parameter ranges far from the transition where the same level of additive noise would not suffice to incur flickering.

\section{Analysis}

We explored two flickering scenarios, and we compared them to a critical slowing down scenario. In the critical slowing down scenario, environmental conditions are gradually changing while the noise magnitude $\sigma$ is constant and relatively weak. We defined weak noise as the level of noise that makes the system (a) to fluctuate around its deterministic oligotrophic equilibrium (red line in Fig. 2, upper left panel) and (b) to shift to the alternative attractor only very close to the actual fold bifurcation.

Contrary to the critical slowing down case, under flickering, noise is strong enough to allow the system occasionally to escape to the alternative basin of attraction (Fig. 2, upper middle panel). At the same time, the likelihood of occasional excursions to the alternative basin and back increases because environmental conditions gradually change and cause the basin of attraction of the low equilibrium to shrink towards the transition (Fig. 2b). In addition, the chances of shifting from the high to the low alternative state also increase as environmental conditions change, due to the fact that disturbances are proportional to the actual levels of $P$ in the lake. Therefore, we refer to this "flickering" behavior as driver-mediated flickering. Under this scenario, the shift to the alternative attractor no longer coincides with the threshold of the fold bifurcation (Guttal and Jayaprakash 2007).

Noise-mediated flickering is another way of incurring occasional jumps between alternative basins of attraction. In the noise-mediated flickering, though, such jumps are caused only by the perturbations themselves, while environmental conditions remain constant (Fig. 2, upper right panel). This means that it is not the driver that changes the stability of the current basin of attraction to allow the system to cross the boundary of the two basins. Instead, for a static basin of attraction, it is the increasing noise magnitude that can tip the system back and forth between the basins of the two alternative attractors (Fig. 2, c1).

In all three scenarios, we derived generic leading indicators from (a) stationary distributions and (b) transient simulation experiments. Stationary distribution experiments allow the estimation of the indicators along a gradient of constant conditions. For each level of environmental conditions, we simulated the model after discarding transients so that the sampled time series are deviations around the underlying deterministic equilibrium for the particular level of environmental conditions. Transient simulation experiments represent a more realistic situation of time series measured in the field: We changed environmental conditions at the same rate with time, and we monitored the resulting time series of $P$ concentration in the lake. Every time step in the time series corresponds to a different environmental level, which represents a realistic scenario where transients are inevitable.

\section{Stationary distributions experiments}

In the critical slowing down and driver-mediated flickering scenarios, we estimated stationary distributions in a 100step gradient of the driver (phosphorus input rate $\alpha$ ) ranging from 0.25 (where the oligotrophic state is the only possible equilibrium) to 0.75 (where the eutrophic state is the only possible equilibrium). At each level of the driver, we ran the model for 250 time steps. We discarded the first 50 time steps to minimize transient effects, and we used the last 200 points to calculate leading indicators: standard deviation, autocorrelation at-lag-1, and skewness. We used a noise intensity $\sigma$ of 0.05 (weak) for the critical slowing down scenario and $\sigma$ of 0.25 (strong) for the driver-mediated flickering scenario.

In the noise-mediated flickering scenario, as we were interested in flickering caused by noise and not by a shrinking attraction basin, we chose a specific level of phosphorus input halfway in the bistability region $(\alpha=0.52528)$, and at that level, we estimated leading indicators for a 100 -step 
Fig. 2 Critical slowing down vs flickering. In the critical slowing down scenario, the system state stays within the basin of attraction of the current state under weak disturbances (upper left panel), while in the flickering scenarios, stronger disturbances can push the system state across the basin of attraction when the basin of attraction shrinks (drivermediated flickering middle panel) or when noise intensity increases (noise-mediated flickering left panel). An example of a typical time series derived from transient simulations under critical slowing down (a), drivermediated flickering (b), and noise-mediated flickering (c) scenarios. Generic leading indicators [variance measured as standard deviation $(a 1, b 1$, c1), autocorrelation at-lag-1 (AR1, a2, b2, c2), and skewness $(a 3, b 3, c 3)]$ were estimated for each scenario within sliding windows of 200 points across the time series (solid red lines denote stable equilibria, dotted red lines unstable equilibria, and red dash dot lines the threshold at which the critical transition to the eutrophic state would occur in the deterministic case)
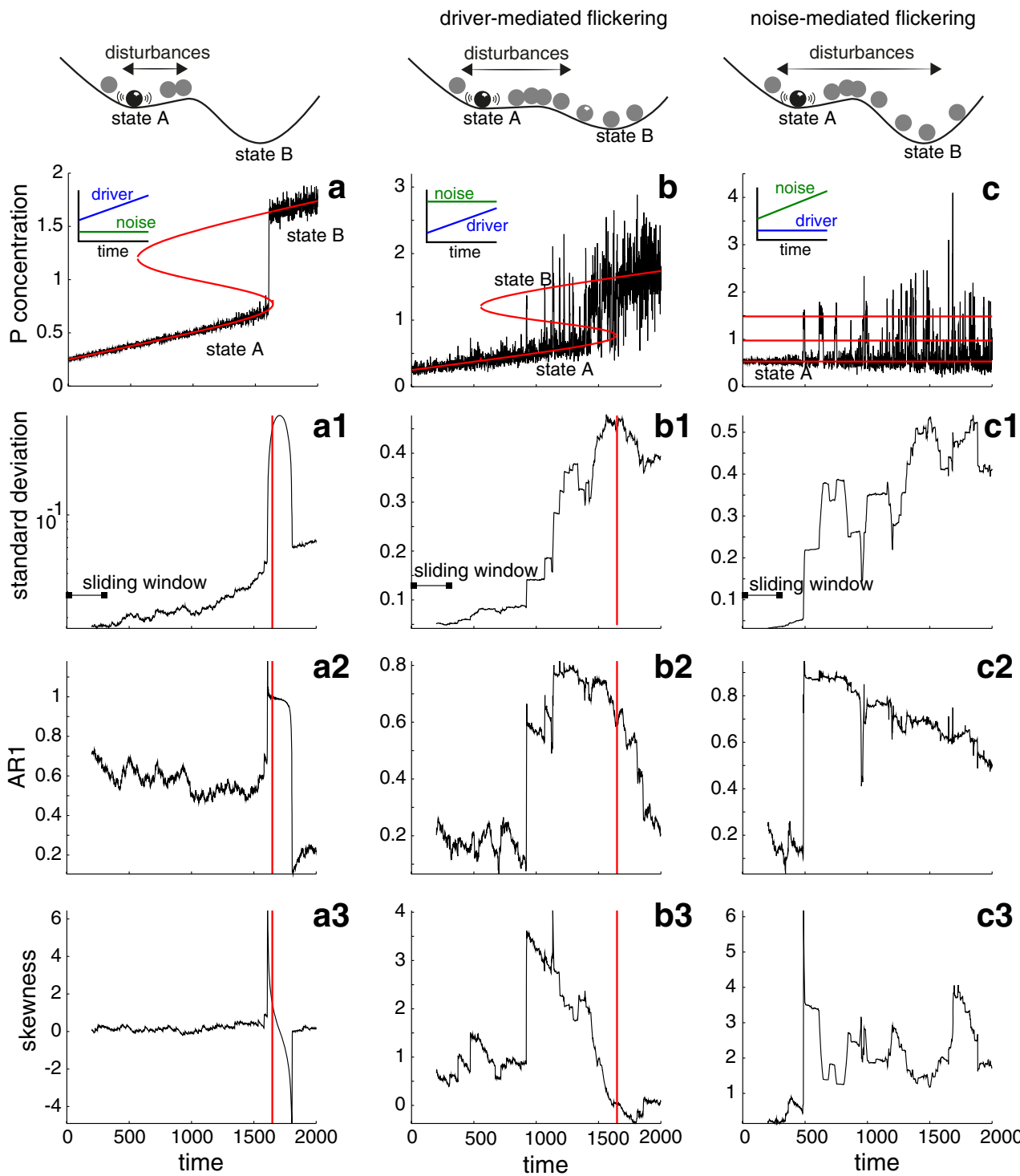

a3
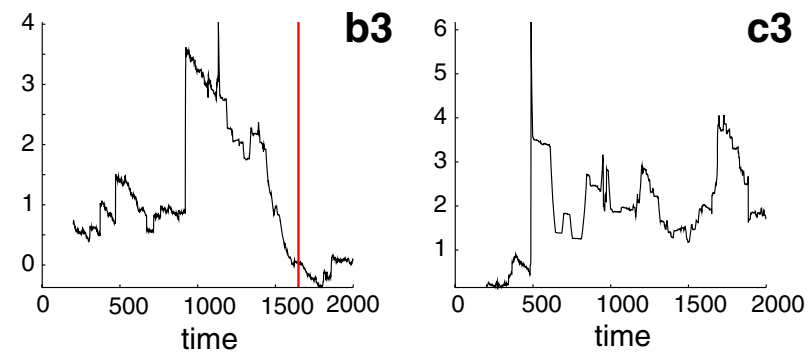

gradient of noise intensity $\sigma$ ranging from 0.05 to 0.5 . Like in the driver-mediated scenario, we discarded the first 50 time steps to minimize transient effects and used only the last 200 points to calculate the indicators. For all three scenarios, we obtained stationary distributions by repeating experiments for 200 Monte Carlo simulations. We reported results as 5, 50, and 95 percentiles for the estimates of the indicators. To check whether the phosphorus input rate at which we performed the analysis affects our results, we repeated the above experiment before the bistability region (phosphorus input rate $\alpha=0.25$ ) and at the onset of bistability $(\alpha=0.3887)$.

\section{Transient simulations experiments}

In the transient simulations experiments, we changed conditions at the same rate with time. In the critical slowing down and driver-mediated scenarios, this meant that we started simulations from the oligotrophic state, and we monitored phosphorus concentration as the input rate progressively increased from 0.25 to 0.75 in 2,000 time steps. In the noise-mediated scenario, we started simulations from the oligotrophic state for the same three above-mentioned levels of phosphorus input rate, and without discarding transients, we monitored phosphorus concentration as the noise intensity progressively increased from 0.05 to 0.5 in 2,000 time steps. In all scenarios, we repeated the same experiment 200 times, and we reported results as 5,50, and 95 percentiles of the leading indicators. We estimated leading indicators within sliding windows of 200 points. Although it has been shown that trends in critical slowing down indicators derived from moving windows depend upon detrending and window-size choices (Dakos et al. 2008, 2012a; Thompson and Sieber 2010; Lenton et al. 2012), we chose a relative 
small window ( $10 \%$ of time series) that enables to track the slow change of the driver or noise magnitude, even though it may produce more erratic patterns in the trends of the indicators (Lenton et al. 2012).

We also used potential analysis (Livina et al. 2010) to reconstruct the potential of the system using the time series only from transient simulations. The idea is based on the stationary probability distribution $p_{\mathrm{s}}(x)$ of a stochastic system $\mathrm{f}(x)$ (Horsthemke and Lefever 2006):

$p_{\mathrm{s}}(x)=N \exp \left[-2 \frac{V(x)}{\sigma^{2}}\right]$

where $N$ is a normalization constant, $\sigma$ the noise intensity, and $V(x)$ the probabilistic potential given by:

$V(x)=-\left[\int^{x} \frac{f(z)}{g^{2}(z)} \mathrm{dz}-\mathrm{n} \frac{\sigma^{2}}{2} \ln \mathrm{g}(\mathrm{x})\right]$

From Eq. 3, it is clear that, if noise is additive $(g(x)=1)$, the probabilistic potential simply coincides with the deterministic potential of the system and is approximated by $V(x) \propto-\log p(x)$ (Ridolfi et al. 2011). We estimated the probabilistic potential by fitting a sliding smoothing kernel function along a time series (Hirota et al. 2011).

All simulations and statistical analyses were performed in MATLAB (v. 7.0.1) and by following the Early Warning Signals Toolbox (http://www.early-warning-signals.org/). We solved the stochastic equations using an EulerMurayama integration method with Ito calculus in 36 integration steps.

\section{Results}

Typically, in a time series where a driver pushed the lake to a eutrophic state (Fig. 2a), critical slowing down caused standard deviation to smoothly rise (Fig. 2, a1), autocorrelation at-lag-1 to increase prior to the transition (Fig. 2, a2), and skewness to peak at the shift (Fig. 2, a3). Comparing these trends to flickering (Fig. 2b, c), the evolution of the indicators estimated within the sliding window was less smooth. Only standard deviation increased slowly before flickering for both driver-mediated (Fig. 2, b1) and noisemediated scenarios (Fig. 2c1), whereas autocorrelation and skewness jumped to high values after the system started to flicker (Fig. 2, b2, b3, c2, c3).

At first sight, little information could be derived when looking at the indicators estimated within sliding windows from single examples of flickering time series (Fig. 2). The irregular trends, however, become clearer when looking at total results from the 200 Monte Carlo realizations (Fig. 3). In the case of the driver-mediated flickering, an increase in phosphorus input rate up to the start of flickering (around
800 time steps) caused a clear rise in standard deviation (Fig. 3, b1), while no apparent change was observed in autocorrelation at-lag-1 (Fig. 3, b2) or in skewness (Fig. 3, b3). After the onset of flickering, the strongest rise in all indicators was documented (Fig. 3, b1-b3). Following the steep rise, all indicators declined mainly due to the disappearance of the lower attractor. The system was not anymore trapped in two distinct basins but wandered around the upper equilibrium: Thus, standard deviation retreated to a lower value, autocorrelation plummeted, and skewness reached values close to 0 (no asymmetry in distributions). Interestingly, skewness peaked and decreased, while variance kept rising similarly to spatial variance and skewness found by Guttal \& Jayaprakash (2009), which suggests that combinations of patterns in the indicators may be more informative for signaling flickering than critical slowing down. Indeed, in the critical slowing down scenario, all indicators clearly increased prior to the transition (Fig. 3, a1-a3). Only autocorrelation decreased at the beginning of the time series due to the increasing noise within the sliding window. This decrease, though, was not observed in the case of the stationary distribution experiments (Fig. A1a2, A2a2, see Electronic Supplementary Material, ESM 1).

Similarly, in the case of the noise-mediated flickering standard deviation gradually increased (Fig. 3, c1), autocorrelation stayed almost constant (Fig. 3, c2), and skewness rose (Fig. 3, c3) for low noise magnitudes. An increase in autocorrelation was observed only when noise was strong enough to push the system towards the unstable equilibrium or even the alternative basin of attraction (around time step 500 when noise magnitude reached 0.16). After this point, the system was clearly spending most of the time between the two basins of attraction and all indicators rose strongly. However, as noise increased further, only autocorrelation decreased (Fig. 3, c2). Skewness remained at a high level (Fig. 3, c3), and standard deviation kept increasing (Fig. 3, c1) following, as expected, the increasing noise magnitude. To a great extent, similar trends were obtained from the stationary distributions (ESM 1 Fig. A1c, A2c).

While it was difficult to infer much over the proximity to a transition from the indicators of a single transient in a flickering system (Fig. 2b, c), potential analysis proved more informative. The reconstructed potentials consistently mapped the dominant underlying attractors (Fig. 4). In both flickering scenarios, potential analysis accurately identified the oligotrophic attractor. At the same time, it provided evidence that the system was visiting the emerging alternative state as shown by the probability distributions computed along the transient time series (Fig. 4). Although the high number of unstable and stable attractors implied a high number of false potential attractors-especially in the noise-mediated scenario (Fig. 4b, c) - the mapping confirmed the existence of at least one alternative eutrophic 
Fig. 3 Total results from 200 transient simulations under critical slowing down (a), driver-mediated flickering (b), and noise-mediated flickering (c) scenarios. Generic leading indicators [variance measured as standard deviation $(a 1, b 1$, c1), autocorrelation at-lag-1 (AR1, a2, b2, c2), and skewness $(a 3, b 3, c 3)]$ were estimated for each scenario within sliding windows of 200 points across the time series. Blue lines represent the median and the range of the blue shade the 5 and 95 percentiles of 200 transient simulations (solid red lines denote stable equilibria, dotted red lines unstable equilibria, and red dash dot lines the threshold at which the critical transition to the eutrophic state would occur in the deterministic case) slowing down prior to transition
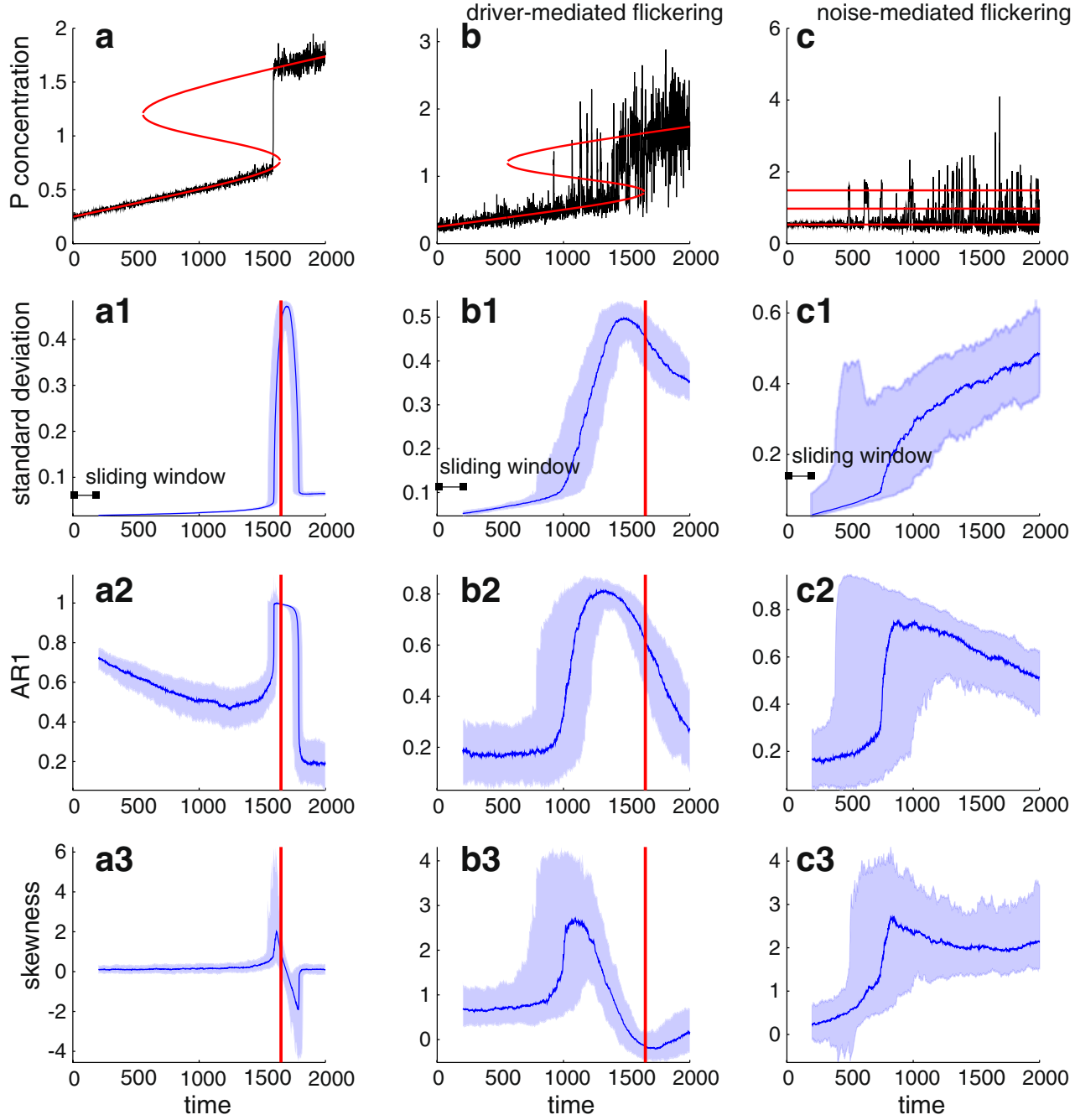

This appears to be especially true for variance, even when estimated within moving windows in single time series regardless of how flickering was induced (Fig. 2). Thus, in flickering time series, contrary to the smooth trends of the indicators under critical slowing down, only trends in variance - and not the erratic and steep changes in autocorrelation and skewness - can most likely be used as indicators of upcoming transitions. Although these findings are in line with earlier observations on the limited detection of critical transitions under strong stochastic conditions (Contamin and Ellison 2009; Perretti and Munch 2012), at the same time, they imply that there may be a range in indicator patterns produced by either critical slowing down, flickering, or extreme stochastic regimes.

Nonetheless, perhaps the most distinct feature of flickering is the fact that it allows us to detect alternative attractors and reconstruct a glimpse of their basins of attraction from probability densities of states (Livina et al. 2010; Dakos et al. 2012a; Scheffer et al. 2012a). By contrast critical slowing down merely signals the loss of resilience in the current flickering. 

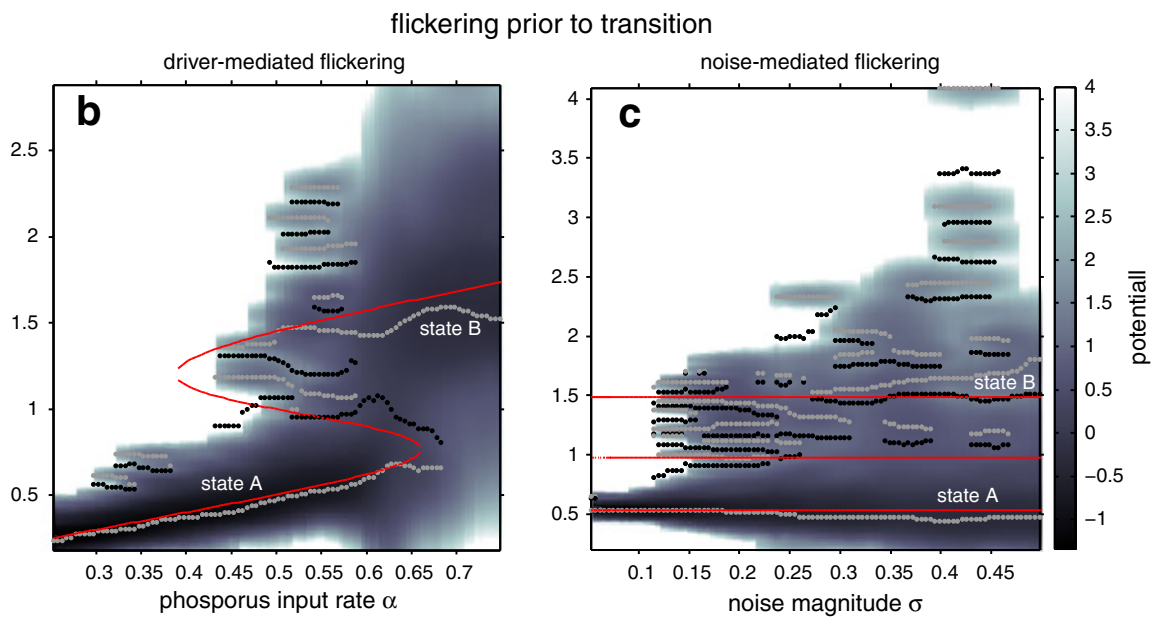

Fig. 4 Reconstructed potential based on a transient simulation under critical slowing down (a), driver-mediated flickering (b), and noisemediated flickering (c) scenarios. Dark gray-blue corresponds to deeper points in the potential landscape, while light gray-blue marks high regions. The lowest values are local minima of the reconstructed potential representing stable attractors (gray dots), while the highest values correspond to local maxima and represent unstable attractors [or the boundaries between alternative basins of the identified local minima (black dots)]. In the critical slowing scenario (a), the two

attractor. In both driver- and noise-mediated scenarios, reconstructing the potential from probability distributions along our simulated time series was an effective way for mapping the presence and location of alternative attractors. However, the identification of the alternative attractors in this case was facilitated by our a priori knowledge of the two underlying equilibria in the model we studied. In real case scenarios, it will be more challenging to determine the appearance of alternative attractors. Overall, combining reconstructed potentials with divergent patterns in the generic leading indicators may be the best way to detect flickering. For example, decreasing autocorrelation and skewness and increasing variance accompanied by weak bimodality in a low-resolution paleo-record prior to eutrophication of the Chinese lake Erhai has been interpreted as evidence for flickering preceding the ultimate transition of the lake to a eutrophic state (Wang et al. 2012).

\section{Prospect}

Clearly, our understanding of these phenomena is still far from complete and perhaps overly simplistic. Deviations from the patterns presented here will be affected by the exact model and the way flickering is induced. For example, in Dakos et al. (2012a,b), flickering takes place in a model that shifts from a high to a low alternative state and shows that the directionality of the shift may have an effect on the patterns in the generic indicators. Given that trends in leading indicators have been shown to depend on the way noise affects dynamics in a system (Brock and Carpenter 2012; alternative attractors are clearly identified and match closely the deterministic equilibria (solid red lines), as well as the onset of the transition. In the driver-mediated flickering scenario, the reconstructed potential quite accurately maps the underlying attractors (b), whereas in the noise-mediated flickering, strong noise creates multiple local minima that are however not persistent (c) (solid red lines denote stable equilibria; dotted red lines mark unstable equilibria of the deterministic system)

Dakos et al. 2012b; Perreti and Munch 2012), it is worthwhile to explore the stochastic conditions at which our conclusions about flickering and its symptoms may fail. For instance, if we assume correlated stochastic conditions affecting model dynamics (Perreti and Munch 2012), it may be difficult to disentangle the effects on autocorrelation as a resilience indicator caused by autocorrelated noise from the effects caused by strong magnitude noise, as we applied here. In such cases, alternative approaches may be useful, like fitting jump-drift-diffusion nonparametric models (Carpenter and Brock 2011) or including an autocorrelated noise term in threshold autoregressive models (Ives and Dakos 2012). Moreover, in addition to externally imposed perturbations, many systems have internal mechanisms that cause a tendency to display cyclic or chaotic dynamics. These dynamics typically interact with external stochasticity to produce a wide range of behaviors (e.g., Wiesenfeld 1993; Bjornstad et al. 2001; Ganopolski and Rahmstorf 2002). For example, although increased variance associated to flickering has been recently identified in high-frequency oscillating time series in patients before the end of an epileptic seizure (Kramer et al. 2012), we are still far from understanding how this range of dynamics will be reflected in leading indicators and reconstructed potentials as an alternative basin of attraction is emerging. Lastly, it has been suggested that one could interpret spatial data from a flickering perspective and in that way identify alternative attractors in space as well (Hirota et al. 2011; Scheffer et al. 2012b). Although much remains to be explored here, some preliminary analysis in a spatially extended model with flickering shows that flickering is 
actually suppressed in space (ESM 1 Online Resource 2). We found that, in strongly connected environments, strong disturbances do not lead to local flickering, but to a synchronized behavior of shifting. Local flickering was possible only for weakly connected systems that lead to gradual transitions (ESM Fig. A6). These results indicate that it is not only the level of disturbance but also the strength of connectivity that determine the behavior of the system (van Nes and Scheffer 2005). The resulting patterns from the leading indicators, however, are very similar to what has been observed in spatial systems with only critical slowing down (Dakos et al. 2011). At the same time, data from the same flickering spatial systems may reveal clearer trends in the leading indicators and in the reconstructed potential than trends from flickering time series. These observations imply that it may be easier than expected to detect critical transitions or alternative attractors in space even under strong stochastic regimes.

In conclusion, the distinction between flickering and critical slowing down is clear in theory, but reality may confront us with a more continuous and complex range of situations. Nonetheless, changes in temporal autocorrelation, variance, and skewness may be useful to alert us to a wide range of phenomena associated to potential upcoming transitions. For flickering systems in particular, exploring probability densities and reconstructing stability landscapes may be the best way for identifying upcoming attractors and detecting approaching transitions.

Acknowledgements We thank Serguei Saavedra and the two anonymous reviewers for helpful comments. We are grateful to Steve Carpenter and Tim Cline for providing us with the planktivorous data for Fig. 1c from the Cascade Project at the University of WisconsinMadison, funded by US NSF. We also thank John Drake for allowing us to use the zooplankton data in Fig. 1d. VD is supported by a Rubicon fellowship from the Netherlands Science Foundation (NWO). EvN and MS acknowledge funding from an Advanced ERC grant awarded to MS.

\section{References}

Bjornstad ON, Grenfell BT, Bjørnstad ON (2001) Noisy clockwork: time series analysis of population fluctuations in animals. Science 293:638-643

Brock WA, Carpenter SR (2012) Early warnings of regime shift when the ecosystem structure is unknown. PLoS One 7:e45586

Carpenter SR, Brock WA (2006) Rising variance: a leading indicator of ecological transition. Ecol Let 9:311-318

Carpenter SR, Brock WA (2010) Early warnings of regime shifts in spatial dynamics using the discrete Fourier transform. Ecosphere 1:art10

Carpenter SR, Brock WA (2011) Early warnings of unknown nonlinear shifts: a nonparametric approach. Ecology 92:2196-2201

Carpenter SR, Ludwig D, Brock WA, Columbia B (1999) Management of eutrophication for lakes subject to potentially irreversible change. Ecol Appl 9:751-771

Carpenter SR, Brock WA, Cole JJ, Kitchell JF, Pace ML (2008) Leading indicators of trophic cascades. Ecol Let 11:128-138
Carpenter SR, Cole JJ, Pace ML, Batt R, Brock WA, Cline T et al (2011) Early warnings of regime shifts: a whole-ecosystem experiment. Science 332:1079-1082

Cimatoribus AA, Drijfhout SS, Livina V, Van der Schrier G (2012) Dansgaard-Oeschger events: tipping points in the climate system. Clim Past Disc 8:4269-4294

Contamin R, Ellison AM (2009) Indicators of regime shifts in ecological systems: what do we need to know and when do we need to know it? Ecol Appl 19:799-816

Dakos V, Scheffer M, Van Nes EH, Brovkin V, Petoukhov V, Held H (2008) Slowing down as an early warning signal for abrupt climate change. PNAS 105:14308-14312

Dakos V, Van Nes EH, Donangelo R, Fort H, Scheffer M (2010) Spatial correlation as leading indicator of catastrophic shifts. Theor Ecol 3:163-174

Dakos V, Kéfi S, Rietkerk M, Van Nes EH, Scheffer M (2011) Slowing down in spatially patterned ecosystems at the brink of collapse. Am Nat 177:E153-E166

Dakos V, Carpenter SR, Brock WA, Ellison AM, Guttal V, Ives AR et al (2012a) Methods for detecting early warnings of critical transitions in time series illustrated using simulated ecological data. PLoS One 7:e41010

Dakos V, Van Nes EH, D’Odorico P, Scheffer M (2012b) Robustness of variance and autocorrelation as indicators of critical slowing down. Ecology 93:264-271

Dansgaard W, Johnsen SJ, Clausen HB, Dahl-Jensen D, Gundestrup NS, Hammer CU et al (1993) Evidence for general instability of past climate from a 250-kyr ice-core record. Nature 364:218-220

Ditlevsen PD, Johnsen SJ (2010) Tipping points: early warning and wishful thinking. Geoph Res Let 37:2-5

Drake JM, Griffen BD (2010) Early warning signals of extinction in deteriorating environments. Nature 467:456-459

Ganopolski A, Rahmstorf S (2002) Abrupt glacial climate changes due to stochastic resonance. Phys Rev Let 88:038501

Guttal V, Jayaprakash C (2007) Impact of noise on bistable ecological systems. Ecol Model 201:420-428

Guttal V, Jayaprakash C (2008) Changing skewness : an early warning signal for regime shifts in ecosystems. Ecol Let 11:450-460

Guttal V, Jayaprakash C (2009) Spatial variance and spatial skewness: leading indicators of regime shifts in spatial ecological systems. Theor Ecol 2:3-12

Hastings A, Wysham DB (2010) Regime shifts in ecological systems can occur with no warning. Ecol Let 13:464-472

Held H, Kleinen T (2004) Detection of climate system bifurcations by degenerate fingerprinting. Geoph Res Let 31:1-4

Hirota M, Holmgren M, Van Nes EH, Scheffer M (2011) Global resilience of tropical forest and savanna to critical transitions. Science 334:232-235

Horsthemke W, Lefever R (2006) Noise-induced transitions: theory and applications in physics, chemistry, and biology. Springer, Berlin

Ives AR, Dakos V (2012) Detecting dynamical changes in nonlinear time series using locally linear state-space models. Ecosphere 3

Kramer MA, Truccolo W, Eden UT, Lepage KQ, Hochberg LR, Eskandar EN (2012) Human seizures self-terminate across spatial scales via a critical transition. PNAS 109:21116-21121

Lenton TM, Livina VN, Dakos V, Van Nes EH, Scheffer M (2012) Early warning of climate tipping points from critical slowing down: comparing methods to improve robustness. Phil Trans R Soc A 370:1185-1204

Livina VN, Kwasniok F, Lenton TM (2010) Potential analysis reveals changing number of climate states during the last 60 kyr. Clim Past 6:77-82

Perretti CT, Munch SB (2012) Regime shift indicators fail under noise levels commonly observed in ecological systems. Ecol Appl 22:1772-1779 
Ridolfi L, D'Odorico P, Laio F (2011) Noise-induced phenomena in the environmental sciences. Cambridge University Press, Cambridge, p 322

Scheffer M, Bascompte J, Brock WA, Brovkin V, Carpenter SR, Dakos V et al (2009) Early-warning signals for critical transitions. Nature 461:53-59

Scheffer M, Carpenter SR, Lenton TM, Bascompte J, Brock W, Dakos V et al (2012a) Anticipating critical transitions. Science 338:344-348

Scheffer M, Hirota M, Holmgren M, Van Nes EH, Chapin FS (2012b) Thresholds for boreal biome transitions. PNAS 109:21384-21389

Strogatz SH (1994) Nonlinear dynamics and chaos with applications to physics, biology, chemistry and engineering. Perseus Books, Reading, p 498

Svensson A, Andersen KK, Bigler M, Clausen HB, Dahl-Jensen D, Davies SM et al (2008) A 60000 year Greenland stratigraphic ice core chronology. Clim Past 4:47-57
Thompson JMT, Sieber J (2010) Climate tipping as a noisy bifurcation: a predictive technique. IMA J Appl Math 76:27-46

Van Nes EH, Scheffer M (2005) Implications of spatial heterogeneity for regime shifts in ecosystems. Ecology 86:1797-1807

Veraart AJ, Faassen EJ, Dakos V, Van Nes EH, Lürling M, Scheffer M (2012) Recovery rates reflect distance to a tipping point in a living system. Nature 481:357-359

Wang R, Dearing JA, Langdon PG, Zhang E, Yang X, Dakos V, Scheffer M (2012) Flickering gives early warning signals of a critical transition to a eutrophic lake state. Nature 492:419-422

Wiesenfeld K (1993) An Introduction to Stochastic Resonance. In: Buchler JR, Kandrup HE (eds) Stochastic processes in astrophysics. The New York Academy of Sciences, New York, pp $13-25$

Wissel C (1984) A universal law of the characteristic return time near thresholds. Oecologia 65:101-107 